relat. vi. et x. p. 74, fig. 2. Quicquid horum sit, majori certitudine diligentissimus ButTnerus, p. 218, avium nidos et ova in Thuringiæ lapicidina tofacea inventa his verbis notatu dignis allegat: Ich bekomme nochmahlige Versicherung," etc.

\title{
MYTILUS SPATHULATUS, A NEW CRETACEOUS SPECIES.
}

\author{
By H. Seeuey, F.G.S.
}

A flint cast of a Mytilus has been obtained from the gravel of Barnwell, near Cambridge, by Mr. Percevall, B.A., Trin. Hall, and entrusted to me for description.

Form elongated and narrow, attenuated anteriorly, with valves deep, and longitudinally striated. The anterior outline of the lips is straight, that of the posterior side a gentle curve, which is somewhat straightened towards the apex. The shell is about three times and a half as long as wide, and widest below the middle. The lateral outline of the valves is lanceolate, the greatest height being in the anterior third.

From the umbones the [subacute] line of inflation ascends, and curves posteriorly, so as to overhang the hinge. It then becomes rounded, and curves into the middle of the shell. The sides descend from it nearly straight. So, on the anterior side of the umbonal end, the sides slope somewhat away from the lips, penthouse-like; while on the posterior side they slope somewhat together, forming a shallow trough. The height of the shell is more than twice its transverse diameter.

The whole is marked with numerous, close, very fine, longitudinal striæ, which appear to be crossed by fine striæ, coincident with the lines of growth, but wider apart. The lips are dentated.

This remarkable form, the first Mytilus yet noticed from the English chalk, must have had a very thin shell, since the faintest exterior ormament seems all preserved on the flint cast.

It is constricted at intervals by rugose bands of growth.

The high valves, laterally compressed form, straight anterior side, and very compressed umbonal end, will readily distinguish this from every other striated cretaceous species. All the forms yet observed in the chalk are striated.

\section{CORRESPONDENCE.}

\section{Spiral Planetary Orbits.}

Dear Sir,-As a regular subscriber to the 'Geologist,' and one interested in its success, I doubt not you will pardon me for a few remarks upon your editorial articles in the last two numbers. 
There are certain views put forward which, unless I am mistaken, are opposed to the known facts of phy sical astronomy, and although my mathematics are rather rusty, $I$ think $I$ am justified in the following criticisms. At p. $\mathbf{4 4 2}$ you speak of effects which changes in the sun's mass would produce in its attraction upon the earth. But would not any matter either dissipated from or deposited on the sun, continue to attract the earth equally before and after dissipation or deposition, the centre of attraction being in every case the centre of gravity of that matter the sun and the earth, and the whole quantity of matter continuing constant. Nor can it be supposed that the position of the centre of gravity could be sensibly altered by such changes.

At p. 443 you refer to Tyndall's expression that the moon "skids the earth." He is considering her tide-generating influence, and shows that she must retard the earth's rotation, but you have spoken, unintentionally no doubt, as if this action had an effect on the orbital motion. Now it is a well-known principle of mechanies, that the motions of rotation and translation are independent of each other; although they may have been originally both given by the same impulse.

Unless I am wrong, there is a mistaken idea which runs through portions of your articles in Nos. 72, 73. You appear to suggest that with a larger extent of orbit there would be greater velocity of the earth in her orbit, and that the effect of a diminished orbit through the action of a resisting medium would be to lessen the velocity in the orbit. One passage in which this idea is presented is this, "if we consider the effects of a higher orbital velocity, we shall find it would give rise probably to a larger extent of orbit."

Now it is usually considered (I may say proved) that the reverse is the case. This may be deduced from Kepler's third law, that the squares of the periodic times of the planets are proportional to the cubes of their mean distances, - a law which is proved, from mechanical considerations, to be true of all cases of planetary motion.

Suppose, for simplicity's sake, that the orbit may be considered a circle for one revolution, which, with the rarity of the cosmical ether and smallness of the ellipticity, is sufficiently true for the purpose.

Expressed symbolically, let $\mathrm{T}$ be the periodic time; $a$, mean distance :

$$
\therefore \mathrm{T}^{2} \propto a^{3}
$$

But in the case of a circular orbit, the velocity (V) is constant:

$$
\begin{array}{r}
\therefore \mathrm{TV}=2 \pi a \\
\mathrm{~T}=\frac{2 \pi a}{\mathrm{~V}} \\
\therefore\left(\frac{2 \pi a}{\mathrm{~V}}\right)^{2} \propto a^{3} \\
\therefore \mathrm{V} \propto \frac{1}{\sqrt{a}}
\end{array}
$$

$\therefore$ As the distance of a planet from the sun is increased, the velocity in the orbit will be diminished, and vice vers $\hat{a}$.

Hence, paradoxical as it might appear, the effect of a resisting medinm by causing the planets to fall towards the sun, and so diminishing their mean distances, is to increase the orbital motion of the heavenly bodies (vide Pratt's 'Mechanical Philosophy,' p. 600). Any changes of the velocity of rotation would be wholly independent of these effects.

Believe me, my dear Sir, fuithfully yours,

Elmstead, Colchester, 2nd January, 1864.

O. Fisher. 